\title{
Some remarks on a family of warped product pointwise semi-slant submanifolds of Kaehler manifold
}

\author{
Ali H. Alkhaldi ${ }^{1}\left(\mathbb{D}\right.$, Akram Ali $^{* 1}$ (1), Mehmet Akif $\mathrm{Akyol}^{2}$ (1) \\ ${ }^{1}$ Department of Mathematics, College of Science, King Khalid University, 9004 Abha, Saudi Arabia \\ ${ }^{2}$ Bingol University, Faculty of Arts and Sciences, Department of Mathematics, 12000, Bingöl, Turkey
}

\begin{abstract}
The purpose of this paper is to provide the complete classifications of the second fundamental form inequality for a warped product pointwise semi-slant submanifold in a Kaehler manifold which was obtained by Şahin [Th. 5.2, Warped product pointwise semi-slant submanifold of Kaehler manifold, Port. Math. 2013] in terms of intrinsic and extrinsic invariants. In this paper, we give some conditions that are not addressed or considered in the cited paper mentioned above. Finally, we provide necessary and sufficient conditions that satisfy inequalities' equalities.
\end{abstract}

Mathematics Subject Classification (2020). 53C40, 53C42, 53B25

Keywords. CR-warped product, warped product, pointwise semi-slant submanifold, modified inequality, Kaehler manifold

\section{Introduction}

Let $N_{1}$ and $N_{2}$ are two Riemannian manifolds with their Riemannian metrics $g_{1}$ and $g_{2}$, respectively and let $f$ be a smooth function defined on $N_{1}$. Then a warped product manifold $M=N_{1} \times{ }_{f} N_{2}$ is a product manifold $N_{1} \times N_{2}$ furnished by a Riemannian metric $g=g_{1}+f^{2} g_{2}[5,18]$. In this case, the smooth function $f$ is a warping function of a warped product manifold. The concept of warped product manifolds is widely used in differential geometry, and many applications of such a warped product manifold are found in numerous situations such as the theory of general relativity [18].

A submanifold $M$ of a Kaehler manifold is said to be a CR-warped product of the form $N_{T} \times{ }_{f} N_{\perp}$ if it is a warped product of a holomorphic submanifold $N_{T}$ and a totally real submanifold $N_{\perp}$. Moreover, Chen [7] claimed that a warped product CR-submanifold of the form $N_{\perp} \times_{f} N_{T}$ of a Kaehler manifold is trivial. In contrast, he also proved the existence of a CR-warped product of the form $N_{T} \times_{f} N_{\perp}$ in a Kaehler manifold and the following relation was established:

\footnotetext{
*Corresponding Author.

Email addresses: ahalkhaldi@kku.edu.sa (A. H. Alkhaldi), akali@kku.edu.sa (A. Ali),

mehmetakifakyol@bingol.edu.tr (M. A. Akyol)

Received: 02.07.2019; Accepted: 07.10.2020
} 
Theorem $1.1([7])$. Let $M=N_{T} \times{ }_{f} N_{\perp}$ be a CR-warped product submanifold in a Kaehler manifold $\widetilde{M}$. Then

(i) The second fundamental form $h$ satisfies the following inequality

$$
\|h\|^{2} \geq 2 n_{2}\|\nabla \ln f\|^{2}
$$

where $\nabla(\ln f)$ is the gradient of $\ln f$ on $N_{T}$ and $n_{2}=\operatorname{dim} N_{\perp}$.

(ii) If the equality holds in (1.1), then $N_{T}$ is totally geodesic in $\widetilde{M}$ and $N_{\perp}$ is a totally umbilical submanifold in $\widetilde{M}$. Moreover, $M$ is a minimal submanifold of $\widetilde{M}$.

In a more general case, Papaghiuc [13] initiated a new class of submanifolds, called a semi-slant submanifold, an extension of CR-submanifold and slant submanifolds. Following the definition of a semi-slant submanifold in a Kaehler manifold, Şahin [14] proved the following result:

Theorem 1.2 ([14]). A warped product semi-slant submanifold $M=N_{T} \times_{f} N_{\theta}$ of a Kaehler manifold is trivial where $N_{T}$ is a holomorphic submanifold and $N_{\theta}$ is a slant submanifold.

By reversing the two factors $N_{T}$ and $N_{\theta}$ of a warped product submanifold, he proved the following result:

Theorem 1.3 ([14]). A semi-slant warped product submanifold of type $M=N_{\theta} \times_{f} N_{T}$ of a Kaehler manifold is trivial.

From Theorem 1.2 and Theorem 1.3, it is seen that a warped product semi-slant submanifold is failed to generalize a CR-warped product submanifold of a Kaehler manifold in the sense of Papaghiuc [13]. Therefore, the class of pointwise slant submanifolds becomes fruitful in Riemannian geometry. A pointwise slant submanifold of almost Hermitian manifolds first appeared in [10] and later characterized by Chen-Garay in [9]. Recently, Şahin [15] discovered the class of pointwise semi-slant submanifold in a Kaehler manifold and discussed associated warped products. He proved the nonexistence of a warped product pointwise semi-slant submanifold as follows:

Theorem 1.4 ([15]). There exists no pointwise semi-slant warped product submanifold of the form $M=N_{\theta} \times_{f} N_{T}$ in a Kaehler manifold such that $N_{\theta}$ is a proper pointwise slant submanifold and $N_{T}$ is a holomorphic submanifold.

By reversing the two factors $N_{T}$ and $N_{\theta}$, the following theorem for the existence of a warped product pointwise semi-slant submanifold in a Kaehler manifold was proved by Şahin [15].

Theorem 1.5 ([15]). Let $M$ be a non-trivial warped product pointwise semi-slant submanifold of the form $N_{T} \times_{f} N_{\theta}$ in a Kaehler manifold. Then

(i) The second fundamental form $h$ satisfies the following inequality

$$
\|h\|^{2} \geq 2 n_{2}\left(\csc ^{2} \theta+\cot ^{2} \theta\right)\|\nabla(\ln f)\|^{2}
$$

where $N_{T}$ is a holomorphic submanifold of dimension $n_{1}$ and $N_{\theta}$ is a proper pointwise slant submanifold of dimension $n_{2}$.

(ii) If the equality in (1.2) holds, then $N_{T}$ is totally geodesic in $\widetilde{M}$ and $N_{\theta}$ is a totally umbilical submanifold in $\widetilde{M}$. Moreover, $M$ is a minimal submanifold of $\widetilde{M}$.

It is noticed that Theorem 1.5 is a generalization of Theorem 1.1 if the pointwise slant function $\theta$ becomes globally constant and substitute $\theta=\frac{\pi}{2}$ in (1.2). Therefore, a warped product pointwise semi-slant submanifold $N_{T} \times{ }_{f} N_{\theta}$ of a Kaehler manifold is generalized 
to a CR-warped product submanifold of the form $N_{T} \times_{f} N_{\perp}$. A Nash embedding theorem [12] implies that every warped product manifold $N_{1} \times{ }_{f} N_{2}$ is isometrically embedded as a Riemannian submanifold in a Euclidean space. Based on Nash theorem and utilizing the Codazzi equation, the following theorem is proved by Chen [8].

Theorem 1.6. Let $\varphi: M=N_{T} \times_{f} N_{\perp} \longrightarrow \widetilde{M}(4 c)$ be isometrically immersed from a CR-warped product submanifold $N_{T} \times_{f} N_{\perp}$ into a complex space form $\widetilde{M}(4 c)$ with a holomorphic constant curvature $4 c$. Then the following inequality is satisfied

$$
\|h\|^{2} \geq 2 n_{2}\left(\|\nabla(\ln f)\|^{2}+\Delta(\ln f)+4 n_{1} n_{2}\right)
$$

where $n_{1}=\operatorname{dim}_{\mathbb{C}} N_{T}$ and $n_{2}=\operatorname{dim} N_{\perp}$. If the equality holds in (1.3), then $N_{T}$ is a totally geodesic submanifold in $\widetilde{M}$ and $N_{\perp}$ is a totally umbilical submanifold in $\widetilde{M}(4 c)$. Moreover, $M$ is a minimal submanifold of $\underset{M}{M}(4 c)$.

Utilizing the Gauss equation instead of the Codazzi equation from the different techniques that used in [16,17], Ali et al.[4] proved a significant theorem for a warped product pointwise semi-slant submanifold, which isometrically immersed into a Kaehler manifold.

Theorem 1.7 ([4]). Let $\varphi: M=N_{T} \times_{f} N_{\theta} \longrightarrow \widetilde{M}$ be an isometrically immersed from a warped product pointwise semi-slant submanifold $N_{T} \times_{f} N_{\theta}$ into a Kaehler manifold $\widetilde{M}$. Then the second fundamental form $h$ satisfies the following:

$$
\|h\|^{2} \geq 2\left(\widetilde{\tau}\left(T M^{n}\right)-\widetilde{\tau}\left(T N_{T}\right)-\widetilde{\tau}\left(T N_{\theta}\right)+n_{2}\|\nabla(\ln f)\|^{2}-n_{2} \Delta(\ln f)\right)
$$

where $\widetilde{\tau}(T M)=\sum_{i<j} \widetilde{K}\left(e_{i} \wedge e_{j}\right)$ and $n_{2}=\operatorname{dim} N_{\theta}$. The equality holds in (1.4) if and only if $N_{T}$ is a totally geodesic submanifold in $\widetilde{M}$ and $N_{\theta}$ is a totally umbilical submanifold in $\widetilde{M}$. Moreover, $M$ is a minimal submanifold of $\widetilde{M}$.

The following theorem is an immediate consequence of Theorem 1.7.

Theorem $1.8([4])$. Let $\varphi: M=N_{T} \times_{f} N_{\theta} \longrightarrow \widetilde{M}(c)$ be an isometrically immersed from a warped product pointwise semi-slant submanifold $N_{T} \times_{f} N_{\theta}$ into a complex space form $\widetilde{M}(c)$ with a holomorphic constant curvature $c$. The following inequality is satisfied

$$
\|h\|^{2} \geq 2 n_{2}\left(\|\nabla(\ln f)\|^{2}-\Delta(\ln f)+\frac{n_{1} c}{4}\right)
$$

where $n_{1}=\operatorname{dim}_{\mathbb{C}} N_{T}$ and $n_{2}=\operatorname{dim} N_{\theta}$. The equality holds in (1.5) if and only if $N_{T}$ is a totally geodesic submanifold in $\widetilde{M}$ and $N_{\theta}$ is a totally umbilical submanifold in $\widetilde{M}(c)$. Moreover, $M$ is a minimal submanifold of $\widetilde{M}(c)$.

Because of Theorems 1.1 and 1.5, a fundamental question arises regarding the converse parts of the second statements in Theorems 1.1 and 1.5, which have not been considered before, i.e.,

Fundamental Question: What can we conclude from the converse part of the statement (ii) of Theorems 1.1 and 1.5, for a CR-warped product submanifold and a warped product pointwise semi-slant submanifold in an arbitrary Kaehler manifold, respectively? Under what condition are these equalities satisfied in Theorems 1.1 and 1.5?

In this paper, we shall modify the inequalities Theorems 1.5 and 1.1 by using the method of Theorem 1.7. We find necessary and sufficient conditions whose equalities of the inequalities (1.1) and (1.2) are satisfied. These reconstructions can be extended to all the inequalities of the second fundamental form in almost Hermitian manifolds. Now using Theorem 1.7, we modify the inequality in Theorem 1.5 of [15] as follows: 
Theorem 1.9. Let $\varphi: M=N_{T} \times_{f} N_{\theta} \longrightarrow \widetilde{M}$ be an isometrically immersed from a warped product pointwise semi-slant submanifold $N_{T} \times_{f} N_{\theta}$ into a Kaehler manifold $\widetilde{M}$. Then the second fundamental form $h$ satisfies the following inequality

$$
\|h\|^{2} \geq 2 n_{2}\left(\csc ^{2} \theta+\cot ^{2} \theta\right)\|\nabla(\ln f)\|^{2}
$$

where $n_{2}=\operatorname{dim} N_{\theta}$. The equality sign holds in (1.6) if and only if $N_{T}, N_{\theta}, M$ are totally geodesic, totally umbilical, minimal submanifolds in $\widetilde{M}$, respectively with the following equality

$$
\widetilde{\tau}(T M)=\widetilde{\tau}\left(T N_{T}\right)+\widetilde{\tau}\left(T N_{\theta}\right)+2 n_{2} \cot ^{2} \theta\|\nabla(\ln f)\|^{2}+n_{2} \Delta(\ln f) .
$$

Remark 1.10. Similarly, Theorem 1.1 is also a special case of Theorem 1.4 as follows: if we choose $\theta=\frac{\pi}{2}$ with globally a constant function $\theta$, then $\cot ^{2} \theta=0$, and $\csc ^{2} \theta=1$. In the same way, taking account of Theorem 1.9, we modify the inequality (1.1) of Theorem 1.1 as follows:

Theorem 1.11. Let $M=N_{T} \times_{f} N_{\perp} \longrightarrow \widetilde{M}$ be an isometrically immersed from a CRwarped product submanifold into a Kaehler manifold $\widetilde{M}$. Then

(i) the second fundamental form $h$ satisfies

$$
\|h\|^{2} \geq 2 n_{2}\|\nabla \ln f\|^{2}
$$

where $\nabla(\ln f)$ is the gradient of $\ln f$ on $N_{T}$ and $n_{2}=\operatorname{dim} N_{\perp}$,

(ii) the equality holds in (1.8) if and only if $N_{T}, N_{\perp}, M$ are totally geodesic, totally umbilical, minimal submanifolds in $\widetilde{M}$, respectively and also satisfies

$$
\widetilde{\tau}(T M)=\widetilde{\tau}\left(T N_{T}\right)+\widetilde{\tau}\left(T N_{\theta}\right)+n_{2} \Delta(\ln f)
$$

where $\Delta$ is the Laplacian on $N_{T}$.

Remark 1.12. The above theorems can be considered as modified versions of Chen-type inequalities for the second fundamental form of a warped product pointwise semi-slant submanifold (see $[2,4,11,16]$ for more detail).

Now, we obtain the next result as an interesting application of Theorem 1.9 if $\widetilde{M}(c)$ is a complex space form with holomorphic constant sectional curvature $c$.

Theorem 1.13. Let $\varphi: M=N_{T} \times_{f} N_{\theta} \longrightarrow \widetilde{M}(c)$ be an isometrically immersed from a warped product pointwise semi-slant submanifold $N_{T} \times_{f} N_{\theta}$ into a complex space form $\widetilde{M}(c)$. Then the following inequality holds

$$
\|h\|^{2} \geq 2 n_{2}\left(\csc ^{2} \theta+\cot ^{2} \theta\right)\|\nabla(\ln f)\|^{2}
$$

where $n_{2}=\operatorname{dim} N_{\theta}$. The equality holds in (1.10) if and only if $N_{T}, N_{\theta}, M$ are totally geodesic, totally umbilical, minimal submanifolds in $\widetilde{M}$, respectively and satisfies

$$
2 \cot ^{2} \theta\|\nabla(\ln f)\|^{2}+\Delta(\ln f)=\frac{n_{1} c}{4}
$$

where $c$ is a holomorphic constant sectional curvature.

Similarly, the following result is a consequence of Theorem 1.7.

Theorem 1.14. Let $\varphi: M=N_{T} \times_{f} N_{\theta} \longrightarrow \widetilde{M}$ be an isometrically immersed from a warped product pointwise semi-slant submanifold $N_{T} \times_{f} N_{\theta}$ into a Kaehler manifold $\widetilde{M}$ such that $N_{T}$ is a compact base without boundary. If the following equality holds

$$
\int_{N_{T} \times\left\{n_{2}\right\}}\left(\left\|h\left(\mathcal{D}, \mathcal{D}^{\theta}\right)\right\|^{2}\right) \mathrm{dV}=\int_{N_{T} \times\left\{n_{2}\right\}}\left(\widetilde{\tau}(T M)-\widetilde{\tau}\left(T N_{T}\right)-\widetilde{\tau}\left(T N_{\theta}\right)\right) \mathrm{dV}
$$

then one of the statements is true for $M$ : 
(i) A warped product pointwise semi-slant submanifold $N_{T} \times_{f} N_{\theta}$ in a Kaehler manifold $\widetilde{M}$ is a CR-warped product.

(ii) A non-trivial warped product pointwise semi-slant submanifold $N_{T} \times_{f} N_{\theta}$ in a Kaehler manifold $\widetilde{M}$ is a simply Riemannian product of $N_{T}$ and $N_{\theta}$.

Similar results shall be presented as corollaries.

\section{Preliminaries}

Let $(\widetilde{M}, g)$ be an almost Hermitian manifold. This means [19] that $\widetilde{M}$ admits a tensor field $J$ of type $(1,1)$ on $\widetilde{M}$ such that, $\forall X, Y \in \Gamma(T \widetilde{M})$, we have

$$
J^{2}=-I, g(X, Y)=g(J X, J Y) .
$$

An almost Hermitian manifold $\widetilde{M}$ is called Kähler manifold if $\left(\tilde{\nabla}_{X} J\right) Y=0, \forall X, Y \in$ $\Gamma(T \widetilde{M})$, where $\tilde{\nabla}$ is the Levi-Civita connection on $\widetilde{M}$.

Let $M$ be isometrically immersed into an almost Hermitian manifold $\widetilde{M}$ with the induced metric $g$. If $\nabla$ and $\nabla^{\perp}$ are the induced Riemannian connections on the tangent bundle $T M$ and the normal bundle $T^{\perp} M$ of $M$, respectively. Then the Gauss and Weingarten equations are given as $\widetilde{\nabla}_{U} V=\nabla_{U} V+h(U, V)$ and $\widetilde{\nabla}_{U} N=-A_{N} U+\nabla_{U}^{\perp} N$, respectively, for every $U, V \in \Gamma(T M)$ and $N \in \Gamma\left(T^{\perp} M\right)$, where $h$ and $A_{N}$ are the second fundamental form and the shape operator, respectively. They are related by $g(h(U, V), N)=g\left(A_{N} U, V\right)$, where $g$ denotes the Riemannian metric on $\widetilde{M}$ as well as the metric induced on $M$.

Now for any $U \in \Gamma(T M)$ and $N \in \Gamma\left(T^{\perp} M\right)$, we put $J U=T U+F U$, and $J N=$ $t N+f N$, where $T U(t N)$ and $F U(f N)$ are tangential and normal components of $J U(J N)$, respectively. If $T$ is identically zero, then a submanifold $M$ is a totally real submanifold. The Gauss equation for a submanifold $M$ is defined as follows:

$$
\begin{aligned}
\widetilde{R}(X, Y, Z, W)= & R(X, Y, Z, W)+g(h(X, Z), h(Y, W)) \\
& -g(h(X, W), h(Y, Z))
\end{aligned}
$$

for any $X, Y, Z, W \in \Gamma(T M)$, where $\widetilde{R}$ and $R$ are the curvature tensors on $\widetilde{M}$ and $M$, respectively. Similarly, let $\widetilde{M}(4 c)$ be a complex space form with a constant holomorphic sectional curvature $4 c$. Then the curvature tensor $\widetilde{R}$ of $\widetilde{M}(4 c)$ is expressed as follows:

$$
\begin{gathered}
\widetilde{R}(X, Y) Z=c(g(X, Z) Y-g(Y, Z) X+g(X, J Z) J Y \\
-g(Y, J Z) X+2 g(X, J Y) J Z) .
\end{gathered}
$$

The mean curvature vector $H$ for an orthonormal frame $\left\{e_{1}, e_{2}, \ldots, e_{n}\right\}$ of tangent space $T M$ on $M$ is defined by

$$
H=\frac{1}{n} \operatorname{trace}(h)=\frac{1}{n} \sum_{i=1}^{n} h\left(e_{i}, e_{i}\right)
$$

where $n=\operatorname{dim} M$. In addition, we set

$$
h_{i j}^{r}=g\left(h\left(e_{i}, e_{j}\right), e_{r}\right), \text { and }\|h\|^{2}=\sum_{i, j=1}^{n} g\left(h\left(e_{i}, e_{j}\right), h\left(e_{i}, e_{j}\right)\right) .
$$

If $J$ preserves any tangent space of $M$, i.e., $J\left(T_{x} M\right) \subseteq T_{x} M$, for each $x \in M$, then $M$ is called holomorphic submanifold. Similarly, if $J$ maps any tangent space of $M$ into normal space, that is $J\left(T_{x} M\right) \subseteq T^{\perp} M$, for each point $x \in M$, then $M$ is a totally real 
submanifold. Except for the holomorphic and totally real submanifolds, other important classes of submanifolds of a Kaehler manifold, determined by the behavior of the tangent bundle of a submanifold under the action of a complex structure $J$, are CR-submanifolds, slant submanifolds, semi-slant submanifolds, pointwise slant submanifolds and pointwise semi-slant submanifolds. The complete classification of such submanifolds can be found in detail $[1,3,9,15]$.

Following Definition 3.1 in [15], let us denote $p$ and $q$ as ranks of complex distribution $\mathcal{D}^{T}$ and pointwise slant distribution $\mathcal{D}^{\theta}$ of pointwise semi-slant submanifold in a Kaehler manifold $\widetilde{M}$. Then, we have the following remarks:

Remark 2.1. $M$ is an invariant if $q=0$ and a pointwise slant if $p=0$.

Remark 2.2. If a slant function $\theta: M \rightarrow R$ is globally constant on $M$ with $\theta=\frac{\pi}{2}$, then $M$ is a CR-submanifold.

Remark 2.3. If a slant function $\theta \in\left(0, \frac{\pi}{2}\right)$, then $M$ is a proper pointwise semi-slant submanifold.

Remark 2.4. If $\mu$ is an invariant subspace under $J$ of normal bundle $T^{\perp} M$, then the normal bundle $T^{\perp} M$ can be decomposed as $T^{\perp} M=F \mathcal{D}^{\theta} \oplus \mu$ for a pointwise semi-slant submanifold.

Now, let $f$ be a differential function defined on $M$. Then the gradient $\nabla f$ is given by

$$
g(\nabla f, X)=X f, \quad \text { and } \nabla f=\sum_{i=1}^{n} e_{i}(f) e_{i} .
$$

Moreover, the Laplacian $\Delta f$ of $f$ is given by

$$
\Delta f=\sum_{i=1}^{n}\left\{\left(\nabla_{e_{i}} e_{i}\right) f-e_{i}\left(e_{i}(f)\right)\right\}=-\sum_{i=1}^{n} g\left(\nabla_{e_{i}} g r a d f, e_{i}\right) .
$$

Similarly, the Hessian of $f$ is defined as:

$$
\Delta f=- \text { Trace }^{f}=-\sum_{i=1}^{n} H^{f}\left(e_{i}, e_{i}\right)
$$

where $H^{f}$ denote the Hessian of a function $f$. In this context, we shall define another Riemannian intrinsic invariant, called a scalar curvature and it is denoted by $\widetilde{\tau}\left(T_{p} M^{n}\right)$ at a point $p \in M^{n}$, that is:

$$
\widetilde{\tau}\left(T_{p} M^{n}\right)=\sum_{1 \leq i<j \leq n} \widetilde{K}\left(e_{i} \wedge e_{j}\right) .
$$

Similarly, the scalar curvature $\widetilde{\tau}\left(L_{k}\right)$ for $k$-plane is given by

$$
\widetilde{\tau}\left(L_{k}\right)=\sum_{1 \leq i \leq j \leq n} \widetilde{K}_{i j} .
$$

The compact manifold $M$ without boundary will be considered, i.e., $\partial M=\emptyset$. Thus, we have the following lemma.

Lemma 2.5 (Hopf's lemma, [6]). Let $M$ be a compact Riemannian manifold and $f$ a smooth function on $M$ such that $\Delta f \geq 0(\Delta f \leq 0)$. Then $f$ is a constant function on $M$.

Additionally, for a compact orientable Riemannian manifold $M$ without boundary, the concept of integration on a manifold, the following formula holds

$$
\int_{M} \Delta f \mathrm{~d} V=0
$$

where $\mathrm{d} V$ denotes the volume of $M[19]$. 
Lemma 2.6 ([5]). Let $M=N_{1} \times{ }_{f} N_{2}$ be a warped product manifold. Then we have

(i) $\nabla_{X} Y \in \Gamma\left(T N_{1}\right)$

(ii) $\nabla_{Z} X=\nabla_{X} Z=(X \ln f) Z$

(iii) $\nabla_{Z} W=\nabla_{Z}^{\prime} W-g(Z, W) \nabla \ln f$

for any $X, Y \in \Gamma\left(T N_{1}\right)$ and $Z, W \in \Gamma\left(T N_{2}\right)$.

The following remarks are consequences of a warped product submanifold.

Remark 2.7. A warped product manifold $M=N_{1} \times_{f} N_{2}$ is a trivial warped product or a simply Riemannian product manifold if the warping function $f$ is constant.

Remark 2.8. The base manifold $N_{1}$ is totally geodesic and the fiber $N_{2}$ is a totally umbilical submanifold of a warped product manifold $M=N_{1} \times_{f} N_{2}$.

Similarly, from unit vector fields $X$ and $Z$ are tangent to $N_{1}$ and $N_{2}$, respectively, we derive

$$
\begin{aligned}
K(X \wedge Z) & =g(R(X, Z) X, Z)=\left(\nabla_{X} X\right) \ln f g(Z, Z)-g\left(\nabla_{X}((X \ln f) Z), Z\right) \\
& =\left(\nabla_{X} X\right) \ln f g(Z, Z)-g\left(\nabla_{X}(X \ln f) Z+(X \ln f) \nabla_{X} Z, Z\right) \\
& =\left(\nabla_{X} X\right) \ln f g(Z, Z)-(X \ln f)^{2}-X(X \ln f) .
\end{aligned}
$$

Let $\left\{e_{1}, \ldots, e_{n}\right\}$ be an orthonormal frame for $M$, then summing up over the vector fields, we have

$$
\sum_{i=1}^{n_{1}} \sum_{j=1}^{n_{2}} K\left(e_{i} \wedge e_{j}\right)=\sum_{i=1}^{n_{1}} \sum_{j=1}^{n_{2}}\left(\left(\nabla_{e_{i}} e_{i}\right) \ln f-e_{i}\left(e_{i} \ln f\right)-\left(e_{i} \ln f\right)^{2}\right)
$$

which implies that

$$
\sum_{i=1}^{n_{1}} \sum_{j=1}^{n_{2}} K\left(e_{i} \wedge e_{j}\right)=n_{2}\left(\Delta(\ln f)-\|\nabla(\ln f)\|^{2}\right) .
$$

The following equality is proved in [8] for an arbitrary warped product submanifold

$$
\sum_{i=1}^{n_{1}} \sum_{j=1}^{n_{2}} K\left(e_{i} \wedge e_{j}\right)=\frac{n_{2} \Delta f}{f}
$$

Combining (2.13) and (2.14), we get

$$
\frac{\Delta f}{f}=\Delta(\ln f)-\|\nabla(\ln f)\|^{2} .
$$

\section{Non-trivial warped product pointwise semi-slant of type $N_{T} \times_{f} N_{\theta}$}

Before proceeding to the modification case, we recall the following result which was proved by Şahin [15]:

Lemma 3.1 ([15]). Let $M=N_{T} \times_{f} N_{\theta}$ be a warped product pointwise semi-slant submanifold in a Kaehler manifold $\widetilde{M}$ such that $N_{T}$ and $N_{\theta}$ are holomorphic and pointwise slant submanifolds of $\widetilde{M}$, respectively. Then the following results are satisfied:

$$
\begin{aligned}
g(h(X, Z), F T W) & =-(J X \ln f) g(Z, T W)-(X \ln f) \cos ^{2} \theta g(Z, W) \\
g(h(Z, J X), F W) & =(X \ln f) g(Z, W)-(J X \ln f) g(Z, T W) \\
g(h(X, Y), F Z) & =0
\end{aligned}
$$

for any $X, Y \in \Gamma\left(T N_{T}\right)$ and $Z, W \in \Gamma\left(T N_{\theta}\right)$.

Theorem 3.2 ([4]). Let $\varphi: M=N_{T} \times_{f} N_{\theta} \longrightarrow \widetilde{M}$ be an isometrically immersed from a warped product pointwise semi-slant submanifold $N_{T} \times{ }_{f} N_{\theta}$ into a Kaehler manifold $\widetilde{M}$. Then $N_{T}$ is a $\varphi$-minimal submanifold of $\widetilde{M}$. 


\section{Proofs of main results}

In this section we give the proof of theorems that we proposed in the introduction and then improve the equality case in Theorem 1.7. First, we prove the following result for later use:

Lemma 4.1. Let $\varphi$ be a $\mathcal{D}^{\theta}$-minimal isometric immersion from a warped product pointwise semi-slant submanifold $N_{T} \times_{f} N_{\theta}$ into a Kaehler manifold $\widetilde{M}$. Then $N_{\theta}$ is a $\varphi$-totally geodesic submanifold.

Proof. Let us assume that the second fundamental forms of $M$ and $\widetilde{M}$ are denoted by $h^{*}$ and $\widetilde{h}$ respectively. Then we define the following

$$
h(Z, W)+h^{*}(Z, W)=\widetilde{h}(Z, W)
$$

for any vector fields $Z, W \in \Gamma\left(T N_{\theta}\right)$. From above equation and Remark 2.8, we conclude that $N_{\theta}$ is a totally umbilical submanifold in $\widetilde{M}$ due to a totally umbilical submanifold in $M$. By using Lemma 2.6 (iii), Eq. (4.1) can be written as:

$$
h(Z, W)=g(Z, W)(\xi+\nabla(\ln f))
$$

where $\xi$ is a vector field tangent to $T M$ and normal to $T N_{\theta}$. Considering $\left\{e_{1}^{*}, \cdots, e_{n_{2}}^{*}\right\}$ as an orthonormal frame for a pointwise slant submanifold $N_{\theta}$. Then by taking summation in (4.2) over the vector fields of $N_{\theta}$, we get

$$
\sum_{i, j=1}^{n_{2}} h\left(e_{i}^{*}, e_{j}^{*}\right)=(\xi+\nabla(\ln f)) \sum_{i, j=1}^{n_{2}} g\left(e_{i}^{*}, e_{j}^{*}\right) .
$$

The left hand side of the above equation identically vanishes due to the $\mathcal{D}^{\theta}$-minimality of $\varphi$. Thus (4.3) gives

$$
n_{2}(\xi+\nabla(\ln f))=0
$$

which implies that $N_{\theta}$ is a nonempty and has the following

$$
\xi=-\nabla(\ln f) .
$$

Inserting (4.4) into (4.2), then we get $h(Z, W)=0$ for every $Z, W \in \Gamma\left(T N_{\theta}\right)$. This means that $N_{\theta}$ is a $\varphi$-totally geodesic.

The following lemma is derived from Lemma 4.1

Lemma 4.2. Let $\varphi: M=N_{T} \times_{f} N_{\theta} \longrightarrow \widetilde{M}$ be an isometrically immersed from a warped product pointwise semi-slant submanifold $N_{T} \times_{f} N_{\theta}$ into a Kaehler manifold $\widetilde{M}$. Then the mixed second fundamental form satisfies

$$
\left\|h\left(\mathcal{D}, \mathcal{D}^{\theta}\right)\right\|^{2}=\left\{\widetilde{\tau}(T M)+n_{2}\|\nabla(\ln f)\|^{2}-\widetilde{\tau}\left(T N_{T}\right)-\widetilde{\tau}\left(T N_{\theta}\right)-n_{2} \Delta(\ln f)\right\}
$$

where $\widetilde{\tau}(T M)=\sum_{i<j} \widetilde{K}\left(e_{i} \wedge e_{j}\right)$ and $n_{2}=\operatorname{dim} N_{\theta}$.

Proof. Assuming the equality in the inequality (1.4), we have

$$
2\left(\widetilde{\tau}(T M)-\widetilde{\tau}\left(T N_{T}\right)-\widetilde{\tau}\left(T N_{\theta}\right)\right)+2 n_{2}\|\nabla \ln f\|^{2}=\|h\|^{2}+2 n_{2} \Delta(\ln f) .
$$


Using the definition of the distributions $\mathcal{D}$ and $\mathcal{D}^{\theta}$, the above equation can be expressed as:

$$
\begin{aligned}
2\left(\widetilde{\tau}(T M)-\widetilde{\tau}\left(T N_{T}\right)-\widetilde{\tau}\left(T N_{\theta}\right)\right) & +2 n_{2}\|\nabla \ln f\|^{2} \\
& =\|h(\mathcal{D}, \mathcal{D})\|^{2}+\left\|h\left(\mathcal{D}^{\theta}, \mathcal{D}^{\theta}\right)\right\|^{2}+2\left\|h\left(\mathcal{D}, \mathcal{D}^{\theta}\right)\right\|^{2} \\
& +2 n_{2} \Delta(\ln f) .
\end{aligned}
$$

According to the orthonormal frame of $\mathcal{D}$ and $\mathcal{D}^{\theta}$, the following equality is obtained by using (2.5) and (4.6)

$$
\begin{aligned}
2\left(\widetilde{\tau}(T M)-\widetilde{\tau}\left(T N_{T}\right)-\widetilde{\tau}\left(T N_{\theta}\right)\right) & +2 n_{2}\|\nabla \ln f\|^{2} \\
& =\sum_{r=1}^{2 m} \sum_{i, j=1}^{2 d_{1}} g\left(h\left(e_{i}, e_{j}\right), e_{r}\right)^{2}+\sum_{r=1}^{2 m} \sum_{i, j=1}^{2 d_{2}} g\left(h\left(e_{i}^{*}, e_{j}^{*}\right), e_{r}\right)^{2} \\
& +2 \sum_{r=1}^{2 m} \sum_{i=1}^{2 d_{1}} \sum_{j=1}^{2 d_{2}} g\left(h\left(e_{i}, e_{j}^{*}\right), e_{r}\right)^{2}+2 n_{2} \Delta(\ln f)
\end{aligned}
$$

However, the equality in (1.4) implies that $N_{T}$ is a totally geodesic submanifold in $\widetilde{M}^{2 m}$ which means that $h\left(e_{i}, e_{j}\right)=0$ for any $1 \leq i, j \leq 2 d_{1}$. Also, $N_{\theta}$ is totally umbilical and it can be written as $h\left(e_{t}^{*}, e_{s}^{*}\right)=g\left(e_{t}^{*}, e_{s}^{*}\right) H$ for any $1 \leq t, s \leq 2 d_{2}$. Moreover, $M$ assumed to be a minimal submanifold of $\widetilde{M}$, then mean curvature vector $H$ of $M$ is equal to zero, i.e., $H=0$, and hence $h\left(e_{t}^{*}, e_{s}^{*}\right)=0$ for every $1 \leq t, s \leq 2 d_{2}$. Applying the proceeding facts in (4.7), we get

$$
\begin{aligned}
2\left(\widetilde{\tau}(T M)-\widetilde{\tau}\left(T N_{T}\right)-\widetilde{\tau}\left(T N_{\theta}\right)\right) & +2 n_{2}\|\nabla \ln f\|^{2} \\
& =2 \sum_{r=1}^{2 m} \sum_{i=1}^{2 d_{1}} \sum_{j=1}^{2 d_{2}} g\left(h\left(e_{i}, e_{j}^{*}\right)+2 n_{2} \Delta(\ln f) .\right.
\end{aligned}
$$

This implies (4.5).

\section{Proof of Theorem 1.9}

The first case (i) was proved by Şahin in [15]. Now, we shall prove (ii). Considering (4.5), we have

$$
\left\|h\left(\mathcal{D}, \mathcal{D}^{\theta}\right)\right\|^{2}+n_{2} \Delta(\ln f)=\widetilde{\tau}(T M)+n_{2}\|\nabla(\ln f)\|^{2}-\widetilde{\tau}\left(T N_{T}\right)-\widetilde{\tau}\left(T N_{\theta}\right) .
$$

Let $\operatorname{dim} N_{T}=n_{1}=2 d_{1}$ and $\operatorname{dim} N_{\theta}=n_{2}=2 d_{2}$, and $N_{\theta}$ and $N_{T}$ are integral manifolds of the pointwise slant distribution $\mathcal{D}^{\theta}$ and the holomorphic distribution $\mathcal{D}$, respectively. Then we choose $\left\{e_{1}, e_{2}, \ldots, e_{d_{1}}, e_{d_{1}+1}=J e_{1}, \ldots, e_{2 d_{1}}=J e_{d_{1}}\right\}$ and $\left\{e_{2 d_{1}+1}=e_{1}^{*}, \ldots, e_{2 d_{1}+d_{2}}=\right.$ $\left.e_{d_{2}}^{*}, e_{2 d_{1}+d_{2}+1}=e_{d_{2}+1}^{*}=\sec \theta T e_{1}^{*}, \ldots, e_{n_{1}+n_{2}}=e_{n_{2}}^{*}=\sec \theta T e_{d_{2}}^{*}\right\}$ to be orthonormal frames of $T M_{T}$ and $T M_{\theta}$, respectively. Also, the orthonormal frames for the normal subbundles $F D^{\theta}$ and $\mu$ are $\left\{e_{n+1}=\bar{e}_{1}=\csc \theta F e_{1}^{*}, \ldots, e_{n+d_{2}}=\bar{e}_{d_{2}}=\csc \theta F e_{1}^{*}, e_{n+d_{2}+1}=\bar{e}_{d_{2}+1}=\right.$ 
$\left.\csc \theta \sec \theta F T e_{1}^{*}, \ldots, e_{n+2 d_{2}}=\bar{e}_{2 d_{2}}=\csc \theta \sec \theta F T e_{d_{2}}^{*}\right\}$ and $\left\{e_{n+2 d_{2}+1}, \ldots, e_{2 m}\right\}$, respectively. Then (4.9) along the components of $F \mathcal{D}^{\theta}$ and $\mu$ can be expressed

$$
\begin{aligned}
\widetilde{\tau}(T M)+n_{2}\|\nabla(\ln f)\|^{2}-\widetilde{\tau}\left(T N_{T}\right)-\widetilde{\tau}\left(T N_{\theta}\right)-n_{2} \Delta(\ln f) \\
=\sum_{r=1}^{2 d_{2}} \sum_{i=1}^{2 d_{1}} \sum_{j=1}^{2 d_{2}} g\left(h\left(e_{i}, e_{j}^{*}\right), \bar{e}_{r}\right)^{2} \\
\quad+\sum_{r=n+2 d_{2}+1}^{2 m} \sum_{i=1}^{2 d_{1}} \sum_{j=1}^{2 d_{2}} g\left(h\left(e_{i}, e_{j}^{*}\right), e_{r}\right)^{2} .
\end{aligned}
$$

Using Lemma 3.1 in the first term of the right hand side, we easily prove the following

$$
\sum_{r=1}^{2 d_{2}} \sum_{i=1}^{2 d_{1}} \sum_{j=1}^{2 d_{2}} g\left(h\left(e_{i}, e_{j}^{*}\right), \bar{e}_{r}\right)^{2}=n_{2}\left(1+2 \cot ^{2} \theta\right)\|\nabla(\ln f)\|^{2} .
$$

Utilizing (4.10) and (4.11), we find

$$
\begin{aligned}
2 n_{2} \cot ^{2} \theta\|\nabla(\ln f)\|^{2}+n_{2} \Delta(\ln f)+ & \left\|h_{\mu}\left(\mathcal{D}, \mathcal{D}^{\theta}\right)\right\|^{2} \\
& =\widetilde{\tau}(T M)-\widetilde{\tau}\left(T N_{T}\right)-\widetilde{\tau}\left(T N_{\theta}\right) .
\end{aligned}
$$

On the other hand, considering the equality in (1.2) such as:

$$
\|h\|^{2}=2 n_{2}\left(\csc ^{2} \theta+\cot ^{2} \theta\right)\|\nabla(\ln f)\|^{2} .
$$

By definition of the squared norm of the second fundamental form along the components of $N_{T}$ and $N_{\theta}$, we derive

$$
\begin{aligned}
2 n_{2}\left(\csc ^{2} \theta+\cot ^{2} \theta\right)\|\nabla(\ln f)\|^{2}= & \sum_{r=1}^{2 m} \sum_{i, j=1}^{2 d_{1}} g\left(h\left(e_{i}, e_{j}\right), e_{r}\right)^{2}+\sum_{r=1}^{2 m} \sum_{i, j=1}^{2 d_{2}} g\left(h\left(e_{i}^{*}, e_{j}^{*}\right), e_{r}\right)^{2} \\
& +2 \sum_{r=1}^{2 d_{2}} \sum_{i=1}^{2 d_{1}} \sum_{j=1}^{2 d_{2}} g\left(h\left(e_{i}, e_{j}^{*}\right), \bar{e}_{r}\right)^{2} \\
& +2 \sum_{r=n+2 d_{2}+1}^{2 m} \sum_{i=1}^{2 d_{1}} \sum_{j=1}^{2 d_{2}} g\left(h\left(e_{i}, e_{j}^{*}\right), e_{r}\right)^{2}
\end{aligned}
$$

The implication from (4.11) is that:

$$
\sum_{r=1}^{2 m} \sum_{i, j=1}^{2 d_{1}}\left\|h_{T}\left(e_{i}, e_{j}\right)\right\|^{2}+\sum_{r=1}^{2 m} \sum_{i, j=1}^{2 d_{2}}\left\|h_{\theta}\left(e_{i}^{*}, e_{j}^{*}\right)\right\|^{2}+2 \sum_{i=1}^{2 d_{1}} \sum_{j=1}^{2 d_{2}}\left\|h_{\mu}\left(e_{i}, e_{j}^{*}\right)\right\|^{2}=0 .
$$

We easily prove that $N_{T}, N_{\theta}$ and $M$ are totally geodesic, totally umbilical and minimal submanifolds of $\widetilde{M}$, respectively by the techniques of Şahin [15]. Thus Eq. (4.15) gives

$$
\left\|h_{\mu}\left(\mathcal{D}, \mathcal{D}^{\theta}\right)\right\|^{2}=2 \sum_{i=1}^{2 d_{1}} \sum_{j=1}^{2 d_{2}}\left\|h_{\mu}\left(e_{i}, e_{j}^{*}\right)\right\|^{2}=0 .
$$

Using the above equation in (4.12), we finally get

$$
2 n_{2} \cot ^{2} \theta\|\nabla(\ln f)\|^{2}+n_{2} \Delta(\ln f)=\widetilde{\tau}(T M)-\widetilde{\tau}\left(T N_{T}\right)-\widetilde{\tau}\left(T N_{\theta}\right)
$$

which implies (1.7). Thus the first part is proved completely.

Conversely, we assume that $N_{T} \times_{f} N_{\theta}$ is a minimal warped product pointwise semislant submanifold of a Kaehler manifold $\widetilde{M}$ such that $N_{T}$ is totally geodesic and $N_{\theta}$ is totally umbilical in $\widetilde{M}$. Then the following equality holds:

$$
\widetilde{\tau}(T M)=\widetilde{\tau}\left(T N_{T}\right)+\widetilde{\tau}\left(T N_{\theta}\right)+2 n_{2} \cot ^{2} \theta\|\nabla(\ln f)\|^{2}+n_{2} \Delta(\ln f) .
$$


Inserting (4.18) into (4.12), we obtain

$$
\left\|h_{\mu}\left(\mathcal{D}, \mathcal{D}^{\theta}\right)\right\|^{2}=0 .
$$

On the other hand, $N_{T}$ is a totally geodesic submanifold in both $M$ and $\widetilde{M}$, we find that

$$
\left\|h_{T}(\mathcal{D}, \mathcal{D})\right\|^{2}=0 \text {. }
$$

From the hypothesis of the previous result in view of Theorem 3.2, it is proved that $N_{T}$ is a minimal submanifold of $\widetilde{M}$. Besides $M$ is assumed to be a minimal submanifold of $\widetilde{M}$, then $M$ is a $\mathcal{D}^{\theta}$-minimal in $\widetilde{M}$. Thus, in view of Theorem 4.1, we obtained the following:

$$
\left\|h_{\theta}\left(\mathcal{D}^{\theta}, \mathcal{D}^{\theta}\right)\right\|^{2}=0 .
$$

Combining (4.20) and (4.21), the equality (ii) holds identically. This completes the proof of the theorem.

It is noted that a CR-warped product of the form $N_{T} \times_{f} N_{\perp}$ in a Kaehler manifold is a special case of a warped product pointwise semi-slant submanifold $N_{T} \times_{f} N_{\theta}$ in the sense of Şahin [15]. Therefore, the following remark is important.

Remark 4.3. If the pointwise slant function $\theta$ becomes globally constant on $M$, then it generalizes to a slant submanifold. For this case, we consider $\theta=\frac{\pi}{2}$ in Theorem 4.1 and Theorem 4.2, then both Theorem 4.1 and Theorem 4.2 are generalized the results for CR-warped products in a Kaehler manifold $\widetilde{M}$. Therefore, the following corollaries hold:

Corollary 4.4. Let $\varphi$ be a $\mathcal{D}^{\perp}$-minimal isometric immersion from a CR-warped product submanifold $N_{T} \times_{f} N_{\perp}$ into a Kaehler manifold $\widetilde{M}$ such that $N_{\perp}$ is a totally umbilical in $\widetilde{M}$. Then $N_{\perp}$ is a $\varphi$-totally geodesic submanifold.

Corollary 4.5. Let $\varphi: M=N_{T} \times_{f} N_{\perp} \longrightarrow \widetilde{M}$ be an isometrically immersed from a CR-warped product submanifold $N_{T} \times_{f} N_{\perp}$ into a Kaehler manifold $\widetilde{M}$. Then the mixed second fundamental is defined by

$$
\left\|h\left(\mathcal{D}, \mathcal{D}^{\perp}\right)\right\|^{2}=\left\{\widetilde{\tau}(T M)+n_{2}\|\nabla(\ln f)\|^{2}-\widetilde{\tau}\left(T N_{T}\right)-\widetilde{\tau}\left(T N_{\perp}\right)-n_{2} \Delta(\ln f)\right\}
$$

where $\widetilde{\tau}(T M)=\sum_{i<j} \widetilde{K}\left(e_{i} \wedge e_{j}\right)$ and $n_{2}=\operatorname{dim} N_{\perp}$.

Remark 4.6. The inequalities (1.6) and (1.8) are more efficient than the inequalities obtained in (1.2) and (1.1) by two reasons: First, we discussed the equality cases completely, which had not been considered in (1.2) and (1.1). Second, taking account of Theorem 1.9 and Theorem 1.11, we directly obtain the relation in terms of a holomorphic constant sectional curvature $c$ for a complex space forms.

\subsection{Applications of Theorem 1.9}

\section{Proof of Theorem 1.13}

Using the same techniques as the second author used in [4], the following is provided

$$
\begin{aligned}
\widetilde{\tau}(T M) & =\frac{c}{8}\left\{n(n-1)+3\left(n_{1}+n_{2} \cos ^{2} \theta\right)\right\} . \\
\widetilde{\tau}\left(T N_{T}\right) & =\frac{c}{8}\left\{n_{1}\left(n_{1}-1\right)+3 n_{1}\right\}, \\
\widetilde{\tau}\left(T N_{\theta}\right) & =\frac{c}{8}\left\{n_{2}\left(n_{2}-1\right)+3 n_{2} \cos ^{2} \theta\right\} .
\end{aligned}
$$

Putting (4.23), (4.24) and (4.25) in Theorem 1.9, we get proof of our results.

On the other hand, Remark 2.2 and Theorem 1.13 give the following 
Corollary 4.7. Let $\varphi: M=N_{T} \times_{f} N_{\perp} \longrightarrow \widetilde{M}(c)$ be an isometrically immersed from a CR-warped product submanifold $N_{T} \times_{f} N_{\theta}$ into a complex space form $\widetilde{M}(c)$. Then

$$
\|h\|^{2} \geq 2 n_{2}\|\nabla(\ln f)\|^{2}
$$

where $n_{2}=\operatorname{dim} N_{\perp}$. The equality holds in (4.26) if and only if $N_{T}, N_{\perp}, M$ are totally geodesic, totally umbilical, minimal submanifolds in $\widetilde{M}$, respectively and also satisfies

$$
\Delta(\ln f)=\frac{n_{1} c}{4}
$$

where $c$ is a holomorphic constant sectional curvature.

\subsection{Compactness of the Laplacian operator on warped product pointwise semi-slant submanifolds of Kaehler manifold}

\section{Proof of Theorem 1.14}

Taking the integration over the compact pointwise semi-slant warped product submanifold in (4.12) with empty boundary, and making use of (2.11), we get

$$
\begin{aligned}
\int_{N_{T} \times\left\{n_{2}\right\}}\left(\left\|h\left(\mathcal{D}, \mathcal{D}^{\theta}\right)\right\|^{2}\right) \mathrm{dV}= & \int_{N_{T} \times\left\{n_{2}\right\}}\left(\widetilde{\tau}(T M)-\widetilde{\tau}\left(T N_{T}\right)-\widetilde{\tau}\left(T N_{\theta}\right)\right) \mathrm{dV} \\
& +2 n_{2} \int_{N_{T} \times\left\{n_{2}\right\}}\left(\cot ^{2} \theta\|\nabla(\ln f)\|^{2}\right) \mathrm{dV} .
\end{aligned}
$$

If the equality (1.12) is satisfied, then from (4.28) we get the following condition

$$
2 \cot ^{2} \theta\|\nabla \ln f\|^{2}=0 .
$$

Therefore, from the above equation, we find two cases such that $\cot ^{2} \theta=0$, or $\|\nabla \ln f\|^{2}=$ 0 .

Case I. Let $\cot ^{2} \theta=0$ which implies that $\cos \theta=0$. From the classification in [15], we conclude that $N_{\theta}$ turns into a totally real submanifold. This completes the proof of (i) in Theorem 1.14.

Case II. If $\|\nabla \ln f\|^{2}=0$, then $\nabla \ln f=0$. Therefore, $\operatorname{grad} \ln f=0$ and hence $f$ is a constant function on $N_{T}$. From Remark 2.7, we conclude that $M$ is a trivial warped product pointwise semi-slant submanifold of a Kaehler form $\widetilde{M}$. The second part (ii) of Theorem 1.14 is completed.

Taking into account (4.23), (4.24) and (4.25), we give the following corollary as a consequence of Theorem 1.14.

Corollary 4.8. Let $\varphi: M=N_{T} \times_{f} N_{\theta} \longrightarrow \widetilde{M}(c)$ be an isometrically immersed from a pointwise semi-slant warped product submanifold $N_{T} \times_{f} N_{\theta}$ into a complex space form $\widetilde{M}(c)$ with compact base $N_{T}$ and suppose that the following equality holds:

$$
\int_{N_{T} \times\left\{n_{2}\right\}}\left(\left\|h_{\mu}\left(\mathcal{D}, \mathcal{D}^{\theta}\right)\right\|^{2}\right) \mathrm{dV}=\int_{N_{T} \times\left\{n_{2}\right\}}\left(\frac{n_{1} n_{2} c}{4}\right) \mathrm{dV} .
$$

Then one of the following statements is true for $M$ :

(i) A pointwise semi-slant warped product submanifold $N_{T} \times_{f} N_{\theta}$ is a CR-warped product isometrically immersed into a complex space form $\widetilde{M}(c)$.

(ii) A non-trivial pointwise semi-slant warped product submanifold $N_{T} \times_{f} N_{\theta}$ into a complex space form $\widetilde{M}(c)$ is a simply Riemannian product of $N_{T}$ and $N_{\theta}$. 
Acknowledgment. The authors thank the referees for their valuable and constructive comments for modifying the presentation of this work. Also, the authors would like to express his gratitude to Dr. Jae Won Lee (Gyeongsang National University, Korea ) for editing and constructive criticism of the manuscript. Finally, the authors extend their appreciation to the deanship of scientific research at King Khalid University for funding this work through research groups program under grant number R.G.P.2/71/41.

\section{References}

[1] A. Ali, and P. Laurian-Ioan, Geometry of warped product immersion of Kenmotsu space forms and its application to slant immersions, J. Geom. Phy. 114, 276-290, 2017.

[2] A. Ali and P. Laurian-Ioan, Geometric classification of warped products isometrically immersed into Sasakian space forms, Math. Nachr. 292, 234-251, 2019.

[3] A. Ali, and C. Özel, Geometry of warped product pointwise semi-slant submanifolds of cosymplectic manifolds and its applications, Int. J. Geom. Methods Mod. Phys. 14, $1750042,2017$.

[4] A. Ali, S. Uddin, and W.A.M. Othman, Geometry of warped product pointwise semislant submanifolds of Kaehler manifolds, Filomat 32 (12), 3771-3788, 2017.

[5] R.L. Bishop, and B. O'Neill, Manifolds of negative curvature, Trans. Amer. Math. Soc. 145, 1-9, 1969.

[6] O. Calin and D.C. Chang, Geometric mechanics on Riemannian manifolds: applications to partial differential equations, Springer Science \& Business Media, 2006.

[7] B.-Y. Chen, Geometry of warped product CR-submanifold in Kaehler manifolds I, Monatsh. Math. 133, 177-195, 2001.

[8] B.-Y. Chen, Another general inequality for warped product CR-warped product submanifold in complex space forms, Hokkaido Math. J. 32, 415-444, 2003.

[9] B.-Y. Chen, and O. Garay, Pointwise slant submanifolds in almost Hermitian manifolds, Turkish J. Math. 36, 630-640, 2012.

[10] F. Etayo, On quasi-slant submanifolds of an almost Hermitian manifold, Publ. Math. Debrecen 53, 217-223, 1998.

[11] A. Mustafa, Geometry of warped product submanifolds of Riemannian manifolds, $\mathrm{PhD}$ thesis, University of Malaya, 2016.

[12] J. Nash, The imbedding problem for Riemannian manifolds, Ann. of Math. 63, 2063, 1956.

[13] N. Papaghiuc, Semi-slant submanifold of Kaehler manifold, An. St. Univ. Al. I. Cuza. Iasi. 40, 55-61, 1994.

[14] B. Şahin, Non-existence of warped product semi-slant submanifolds of Kaehler manifolds, Geom. Dedicata 117, 95202, 2006.

[15] B. Şahin, Warped product pointwise semi-slant submanifold of Kaehler manifold, Port. Math. 70, 251-268, 2013.

[16] M.M. Tripathi, Improved Chen-Ricci inequality for curvature-like tensors and its applications, Diff. Geom. Appl. 29, 685-698, 2011.

[17] M. M. Tripathi, C-totally real warped product submanifolds, An. St. Univ. Al. I. Cuza. Iasi 58 (2), 417-436, 2012.

[18] B. Ünal, Multiply Warped Products, J. Geom. Phy. 34 (3-4), 287-301, 2000.

[19] K. Yano and M. Kon, Structures on manifolds, World Scientific, 1984. 